\title{
Es lohnt sich, mit dem Rauchen aufzuhören
}

Fragestellung: Ziel dieser Studie war es zu untersuchen, ob Rauchen nach Diagnosestellung einer Multiplen Sklerose (MS) einen Einfluss auf den Zeitpunkt bis zum Übergang in die sekundär chronische Verlaufsform (SPMS) hat.

Hintergrund: Rauchen ist ein etablierter Risikofaktor für MS. Es ist jedoch nicht klar, ob das Rauchen nach der Diagnose schädlich ist.

Patienten und Methodik: Im Rahmen einer Querschnittsstudie wurden 728 Patienten aus dem nationalen schwedischen MSRegister mit bestätigter MS nach den McDonald-Kriterien eingeschlossen, die zum Zeitpunkt der Diagnose rauchten. Als Kontrolle dienten 1.012 nichtrauchende MS-Patienten dieses Registers. Der Raucherstatus wurde jahresweise mittels Fragebogenerhebung ermittelt und galt als positiv bei täglich mindestens einer Zigarette. Primärer Endpunkt war die Zeit von Diagnosestellung bis zur SPMS-Konversion, gemessen in Lebensalter. Dabei dienten der

Ramanujam R, Hedström AK, Manouchehrinia A et al. Effect of smoking cessation on mutiple sclerosis prognosis. JAMA Neurol 2015; 72: $1117-23$ Raucherstatus sowie das Geschlecht, das Alter bei Diagnosestellung, der Genuss von Schnupftabak und Rauchen vor der Diagnosestellung als zeitabhängige Kovariaten in einem AFT-Modell; hierbei handelt es sich um ein statistisches Überlebenszeitmodell unter der Annahme, dass die Kovariaten das Erreichen des Endpunktes beschleunigen oder verzögern.

Ergebnisse: Mittels optimierten AFT-Modell ließ sich zeigen, dass jedes zusätzliche Raucherjahr nach der Diagnose die Zeit bis zur SPMS-Konversion um 4,7\% beschleunigt (Beschleunigungsfaktor 1,047; $95 \%$-Konfidenzintervall [KI] 1,023 - 1,072; $\mathrm{p}<0,001)$. Mittels Kaplan-Meier-Kurve wurde gezeigt, dass jene Patienten, die weiterhin kontinuierlich rauchten, im Median im Alter von 48 Jahren die Konversion zur SPMS durchmachten verglichen mit einem Alter von 56 Jahren bei denjenigen, die nach der Diagnose mit dem Rauchen aufhörten. Die SPMS-Konversionrate war bei Nichtrauchern und fortbestehenden Rauchern nicht unterschiedlich.

Schlussfolgerungen: Diese Studie liefert Hinweise darauf, dass das Rauchen mit einer Beschleunigung der Zeit bis zur SPMSDiagnose assoziiert ist und dass diejenigen, die mit dem Rauchen aufhören, einen Benefit haben. Daher schlagen die Autoren vor, dass Patienten mit MS geraten werden sollte, das Rauchen aufzugeben, sobald die MS-Diagnose gestellt worden ist nicht nur, um die Gefahren für Begleiterkrankungen zu verringern, sondern auch um das Risiko einer MS-bedingten Behinderung zu reduzieren.

\section{- Kommentar von Til Menge, Düsseldorf}

\section{MS und Rauchen gehören nicht zusammen!}

Diese Studie weiß nicht ganz genau, wo sie mit uns hin will. Zum einen zeigt eine Kaplan-Meier-Kurve, dass der Raucherstatus bei Diagnosestellung keinen Einfluss auf das Alter bei SPMSKonversion hat. Auf der anderen Seite sind die Daten aber eindrücklich, dass Weiterrauchen zu einer knapp $5 \%$ igen jährlichen Beschleunigung der Konversion zur SPMS führt, was im Studiensetting im Schnitt acht Jahren entsprach.

Methodisch anzumerken ist jedoch, dass weder eine Definition der notorisch schlecht definierten SPMS angegeben wurde noch erläutert wurde, wer den Wechsel von der schubförmigen in die sekundär progrediente Verlaufsform diagnostizierte. Des Weiteren gab es lediglich die beiden Kategorien Raucher und Nichtraucher, und schon Patienten, die nur eine Zigarette am Tag rauchten, galten als Raucher. Das Ausmaß des Rauchens hingegen wurde nicht berücksichtigt. Dabei hatte die Gruppe der Weiterraucher sogar eine geringfügig höhere Pack-Year Last (12,97 PY vs. 11,65 PY), also vor der MSDiagnose mehr geraucht, als jene, die mit dem Rauchen aufgehört hatten. Die Gruppe der Weiterraucher hatte noch einen Nachteil: Sie begann im Schnitt erst ein Dreivierteljahr später als die Exraucher mit einer krankheitsmodifizierenden Therapie - nachteilig, weil eine verzögerte Frühtherapie prognostisch ungünstig für eine SPMS-Konversion sein kann.
Alles in allem aber werden die Daten zu den klinischen Verläufen gut von den bisher veröffentlichten Studien zu Rauchen und MS validiert, von denen eine bereits 2013 hier vorgestellt wurde [1]. Die Kernaussage hat also Bestand: MS und Rauchen gehören nicht zusammen! Patienten, die über eine krankheitsmodifizierende Therapie hinaus positiv auf die MS einwirken wollen, sollten mit dem Rauchen aufhören.

\footnotetext{
Referenz:

1. INP 2013; Seite 34 [Manouchehrinia A et al. Brain 2013; 136; 2298-304]
}

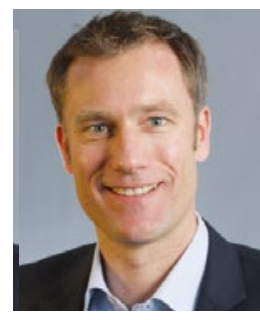

PD Dr. med. Til Menge, Düsseldorf

Leitender Oberarzt am Zentrum für Neurologie und Neuropsychiatrie; LVR-Klinikum Düsseldorf E-Mail:menge@uni-duesseldorf.de 